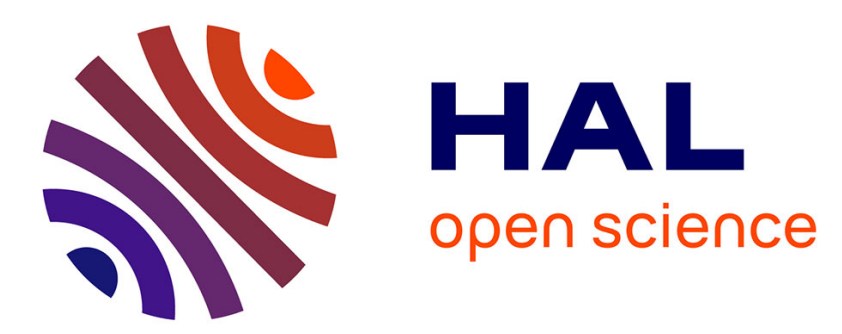

\title{
Experimental signatures of extreme optical fluctuations in lumped Raman fiber amplifiers
}

\author{
Kamal Hammani, Christophe Finot
}

\section{To cite this version:}

Kamal Hammani, Christophe Finot. Experimental signatures of extreme optical fluctuations in lumped Raman fiber amplifiers. Optical Fiber Technology, 2012, 18 (02), pp.93-100. 10.1016/j.yofte.2012.01.003 . hal-00644860

\section{HAL Id: hal-00644860 \\ https://hal.science/hal-00644860}

Submitted on 25 Nov 2011

HAL is a multi-disciplinary open access archive for the deposit and dissemination of scientific research documents, whether they are published or not. The documents may come from teaching and research institutions in France or abroad, or from public or private research centers.
L'archive ouverte pluridisciplinaire HAL, est destinée au dépôt et à la diffusion de documents scientifiques de niveau recherche, publiés ou non, émanant des établissements d'enseignement et de recherche français ou étrangers, des laboratoires publics ou privés. 


\title{
Experimental signatures of extreme optical fluctuations in lumped Raman fiber amplifiers
}

\author{
Kamal Hammani * and Christophe Finot \\ Laboratoire Interdisciplinaire Carnot de Bourgogne, UMR 5209 CNRS-Université de \\ Bourgogne, 9 avenue Alain Savary, BP 47870, 21078 Dijon Cedex, France \\ * Corresponding author: \\ E-mail address: kamal.hammani@u-bourgogne.fr \\ Tel.: +33 380395983
}

\begin{abstract}
:
In this work, we experimentally investigate several temporal and spectral methods to highlight extreme fluctuations which can develop during the Raman amplification of an ultrashort pulse train. Forward and backward pumping schemes are compared to dual pass configurations.
\end{abstract}

Keywords: Fiber optics; Raman amplifiers; extreme statistics 


\section{Introduction}

The study of extreme statistics in nonlinear fiber optics is a topic that has recently stimulated much attention. Following the pioneering work of Solli and coworkers [1] that have reported the observation of statistics analogous to hydrodynamic rogue waves in the formation of optical supercontinuum, most of the initial works have focused on the passive propagation of picosecond pulses in photonic crystal fibers [2]. Those different studies carried out in the anomalous dispersion regime have highlighted the specific role played by modulation instability, Akhmediev breathers $[3,4]$ as well as Raman frequency shifted solitons that are also affected by third-order dispersion and resulting pulse-to-pulse interactions [5, 6]. However, observation of statistics that strongly deviate from a Gaussian distribution is absolutely not restricted to supercontinuum generation and can also be observed in telecommunication applications in the context of transmissions [7-9], Raman fiber lasers [10, 11] as well as discrete amplification [12, 13]. More precisely, in this latest field, it has been shown that under certain conditions, statistical distribution of an amplified signal can be strongly reshaped during the amplification process. Examples of this deleterious degradation have been discussed for parametric amplifiers [12, 14, 15] and for Raman amplifiers [13, 16, 17]: in both cases, a quasi-instantaneous gain and a low walk-off between the signal and a partially coherent pump lead to a dramatic transfer of fluctuations from the pump to the signal in a co-propagating configuration. In a previous work, we have numerically and analytically described the evolution of a continuous signal in a copropagating amplifier in the presence of a low walk-off and pump depletion [17]. We have also shown that an adequate frequency shifted optical bandpass filter can isolate the most extreme structures [16]. 
In this contribution, we focus our attention on experimental results outlining the drastic consequences of the transfer of intensity fluctuations from the pump to a pulsed signal in a lumped Raman amplifier. Indeed, due to their scarcity and their high amplitude, characterization of the impact of extreme statistics remains a tricky task. Contrary to widespread quantitative techniques such as the measurement of the optical noise signal ratio (OSNR) or the relative intensity noise (RIN) [18], we have chosen to present here a set of alternative qualitative methods that show the emergence of extreme fluctuations in a $10 \mathrm{GHz}$ pulse stream. More precisely, this paper will successively present statistical records using photodiodes, optical auto/cross- correlations, RF spectrum and optical spectrum. Forward and backward pumping schemes will be compared to dual-pass configurations.

\section{Experimental Setup}

Our experimental setup is sketched in Fig. 1a. An actively mode-locked erbium-doped fiber laser delivers a train of $2.9 \mathrm{ps}$ pulses at a repetition rate of $10 \mathrm{GHz}$ and with an initial average power of $10 \mathrm{~mW}$ (corresponding to a peak power of $\sim 300 \mathrm{~mW}$ ) at a wavelength of $1550 \mathrm{~nm}$. Let us note that our previous works $[13,16]$ or $[19]$ involved much lower repetition rates as the signal source was a $\mathrm{MHz}$ passively mode-locked fiber laser. The pump is delivered by a Raman continuous wave fiber laser centered at $1455 \mathrm{~nm}$ with an average power up to $2 \mathrm{~W}$. An essential point is that this pump is a partially incoherent wave ( $\sim 20 \mathrm{GHz}$ of spectral width) that exhibits high contrast, fast intensity fluctuations [19]. Indeed, its intensity autocorrelation signal (plotted in Fig. 1b) presents a characteristic contrast of one half $[13,20]$ with a temporal width of 25 ps. This temporal duration is a characteristic time of the shortest pump fluctuations and is much larger than the picosecond signal to be amplified while being shorter than the temporal separation between two successive pulses. 
The amplification is performed in a 1-km long highly nonlinear fiber (HNLF). The low normal dispersion $\left(0.7\right.$ ps. $\left.\mathrm{km}^{-1} \cdot \mathrm{nm}^{-1}\right)$ enables us to avoid solitonic or modulation instability effects. The high nonlinearity $\left(10 \mathrm{~W}^{-1} \cdot \mathrm{km}^{-1}\right)$ leads to a high Raman gain coefficient whereas linear losses and third-order dispersion are limited $\left(0.70 \mathrm{~dB} \cdot \mathrm{km}^{-1}\right.$ and $0.01 \mathrm{ps} . \mathrm{nm}^{-2} \cdot \mathrm{km}^{-1}$ respectively at $1550 \mathrm{~nm}$ ). We have compared several pumping schemes: backward pumping (Fig. 1a1), forward pumping (Fig. 1a3) and two bi-directional pumping (Figs 1a2 and 1a4) relying on the use of optical circulators. Moreover, several pump powers ranging from 500 to $1500 \mathrm{~mW}$ have been tested, leading to integrated gains from 5 to $15 \mathrm{~dB}$ in single pass configurations and gains from 10 to $22 \mathrm{~dB}$ in dual-pass configurations.

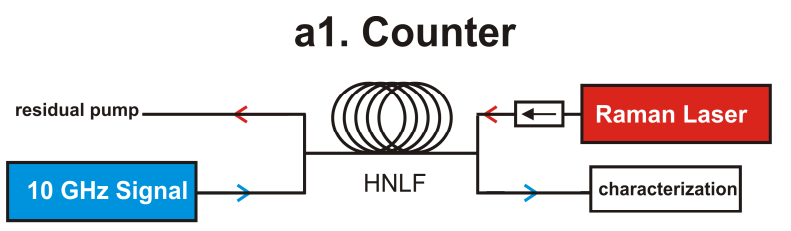

a3. Co

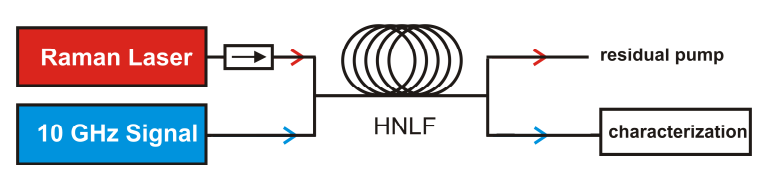

a2. Counter + Co

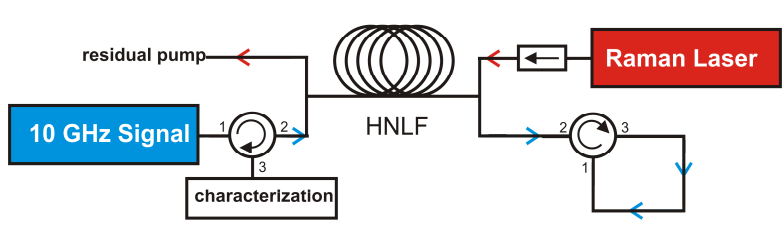

a4. Co + Counter

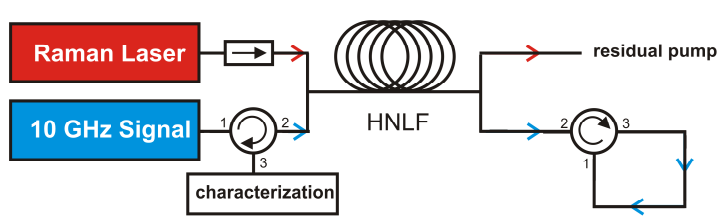

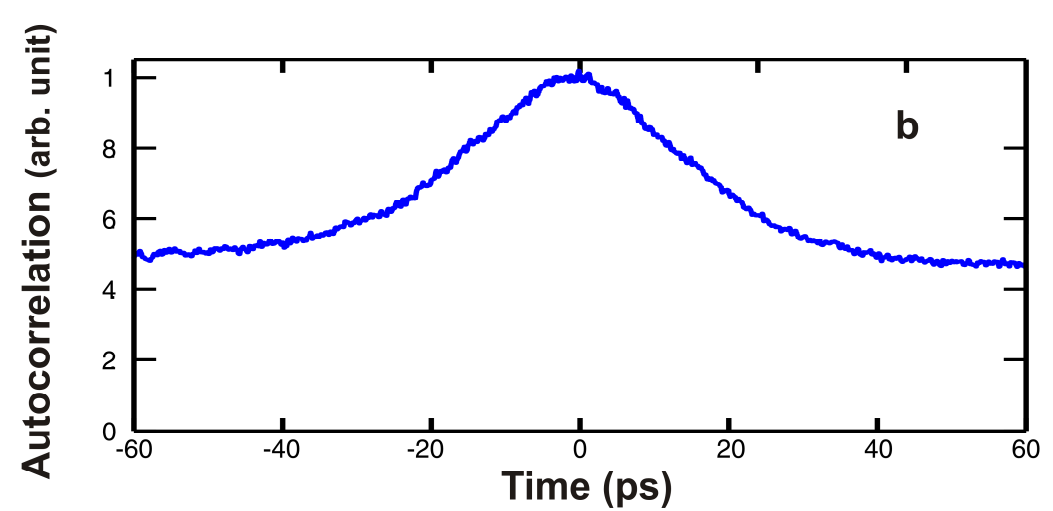

Figure 1: (a1-a4) Various experimental setups under investigation (b) Intensity autocorrelation of the pump. 
Several characterizations of the optical properties of the amplified signal have been carried out. In the temporal domain, we have recorded the output pulse train and its associated statistics thanks to a high speed digital sampling oscilloscope (Tektronix TDS CSA 8200) combined with a photodiode having a $30 \mathrm{GHz}$ bandwidth. We have complemented our study by using an optical intensity autocorrelator (Femtochrome, FR-103HS) to investigate pulse-to-pulse fluctuations. Radio frequency spectra as well as optical spectra were also recorded and further insights on the spectral stability were gained by mapping the spectral evolution into the temporal domain.

\section{Oscilloscope observations and statistical records}

The most intuitive and straightforward way to explore and evaluate signal variation at the output of the Raman amplifier is the direct observation on a high-speed oscilloscope. Indeed, the eyediagram enables us to visualize the variations of the signal level in the various experimental configurations. Given the finite bandwidth of the electronics under use (typically $30 \mathrm{GHz}$, much below the optical bandwidth of our pulses), we do not resolve the details of the temporal intensity profile of the picosecond pulses. Therefore, we do not monitor rigorously the peak power of the pulses, but rather the fluctuations of their energy. As a first approximation and given the fact that the amplified pulses do not exhibit any pulse splitting in our normally dispersive fiber, we can however consider that those energy fluctuations are directly proportional to peak power variations [21].

The choice of the pumping configuration has a strong impact on the amplifier performance as can be readily noticed in Fig. 2. When the signal and the pump counter-propagate in the fiber, the output pulse train exhibits a low level of fluctuations (Fig. 2b) and maintains a quality comparable to the initial mode-locked signal (Fig. 2a). On the contrary, in a copropagating 
scheme, the stability of the output pulse train is drastically affected (Fig. 2c) and striking fluctuations are observed. Similar conclusions can be drawn in bidirectional configurations (Fig 2d).

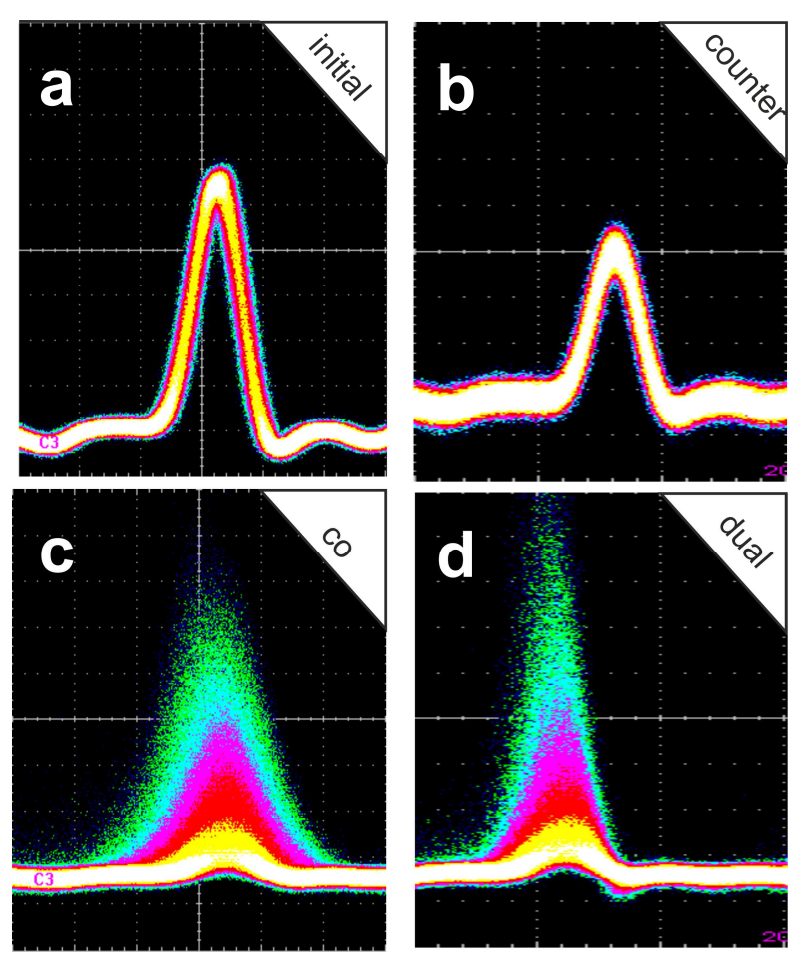

Figure 2: Eye diagrams in several cases: a) initial mode-locked laser, b) counter-propagating configuration, c) co-propagating configuration d) dual pass configuration.

We took advantage of the statistical functions embedded in the oscilloscope to get more quantitative data on the statistical distribution of the pulse energy fluctuations (Fig. 3). Results obtained for different pumping schemes (with a pump power of $1 \mathrm{~W}$ ) are summarized in Fig. 3a and confirm the previous qualitative conclusions. In a backward pumping scheme, the statistical distribution of the pulse energy is quite narrow and can be compared to the initial pulse distribution. It heavily contrasts with the results recorded in a co-propagating scheme where the output statistical distribution presents heavy tails [13]. Events with pulse energy more than 30 times the median energy have indeed been recorded, illustrating the completely unusual amplitude of those fluctuations. Dual pass configurations exhibits slightly less degraded results, 
the most extreme events being less frequent compared to the single pass co-propagating scheme.

This can be mainly explained by the impact of gain saturation of the amplifier that ultimately limits the maximum energy that can be reached.

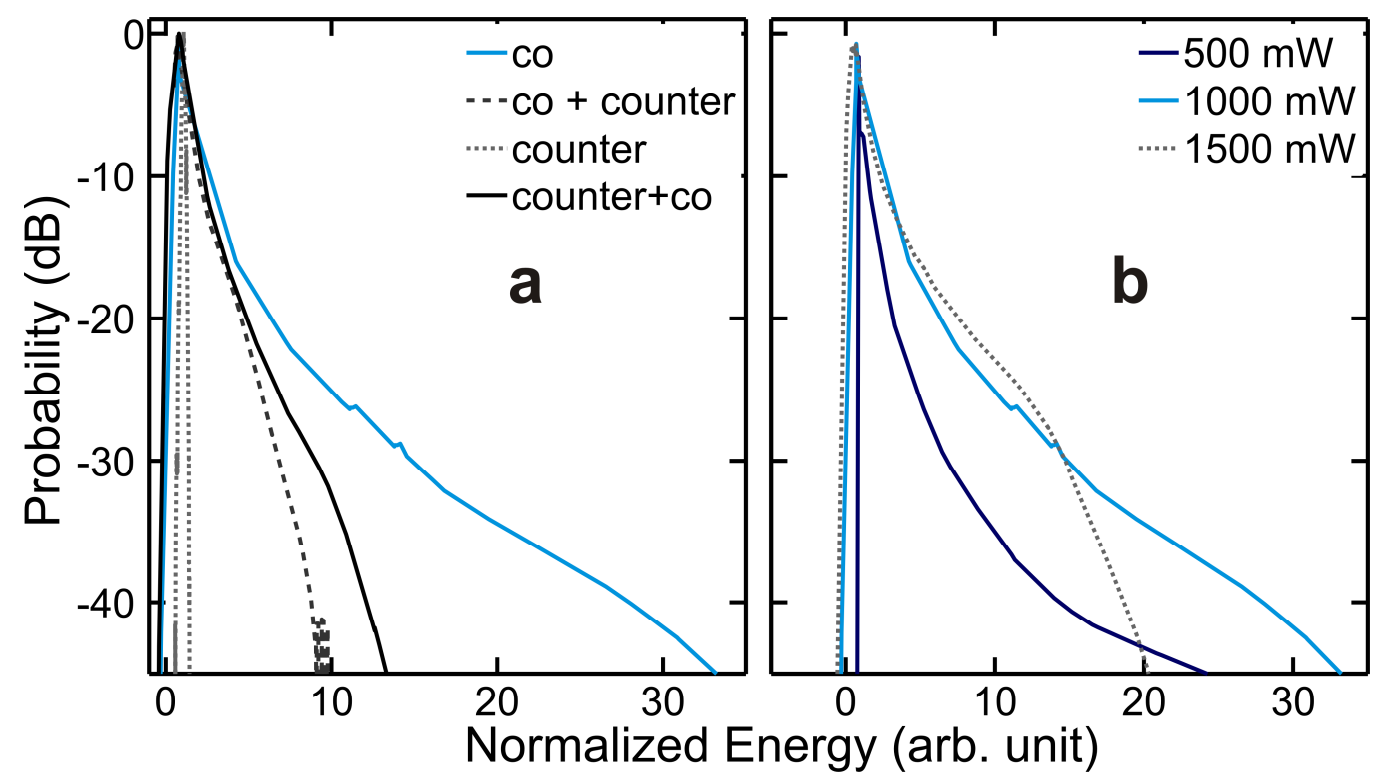

Figure 3: Probability distribution of the amplified signal energies. The results are normalized by the median value. (a) Impact of the pumping configurations for a fixed initial pump power of $1000 \mathrm{~mW}$. Results obtained in a counter-propagating scheme (dotted grey line) are compared to results recorded in co-propagating, co + counter-propagating and counter + co-propagating schemes (solid light blue line, dashed black line, solid black line respectively). (b) Impact of the pump power in a co-propagating configuration. Results obtained for a $1 \mathrm{~W}$ pump power (solid light blue line) are compared with results obtained for pump powers of 0.5 and $1.5 \mathrm{~W}$ (solid dark blue line and dotted grey line respectively).

We have also investigated in more details the influence of the pump power in a forward pumping scheme (Fig. 3b). Increasing the pump power impacts the tail of the statistical distribution by increasing the level of fluctuations: measurements carried out for pumping values between 500 and $1500 \mathrm{~mW}$ show that the probability of observing an event between 2 and 12 times the median energy increases with pump power. However, for larger gains and given the high repetition rate of the signal to be amplified, pump depletion effects and gain saturation become important, which leads to an upper limit of the pump energy and rapidly falling 
probability. Such a behavior is in full agreement with the numerical and analytical conclusions discussed in a previous article dealing with a continuous seed [17].

\section{Optical correlations}

The electrical bandwidth of our oscilloscope is not high enough to resolve the details of the pulse structure and to get access to the duration of the picosecond pulses. Even if optical sampling oscilloscopes with a $\mathrm{THz}$ bandwidth [22] are nowadays available to resolve accurately the temporal profile of periodic pulse trains, we have based here our measurements on a standard, widely spread intensity autocorrelator. Intensity autocorrelations have already been used in previous works in Raman or parametric amplifiers [12, 13, 17] using a continuous seed, providing indirect evidence of the existence of spikes of light.

Results obtained with a 10-GHz picosecond pulse train are plotted in Fig. 4. For single-pass amplifiers, no major modification of the temporal intensity autocorrelation can be noticed compared to the initial pulses. Similar output durations can be recorded in co- and counterpropagating configurations. This has to be linked to the weak dispersion of the highly nonlinear fiber that leads to a dispersion length (defined as the distance for which the initial pulse broadens by a factor 2 due to dispersion [23]) above $5.5 \mathrm{~km}$, which is much higher than the fiber length. However, let us note that the output duration obtained in the forward pumping scheme is slightly higher. Indeed, in this configuration, the pulse experiences more self-phase and cross-phase modulation [16, 24] (this point will be experimentally discussed in section 6 of this paper) so that the expanded spectrum leads to a slightly enhanced dispersion-induced temporal broadening.

Differences are much more marked when comparing the results obtained in dual-pass configurations. More precisely, the scheme where forward pumping is followed by backward 
pumping leads to significantly broadened output pulses. Such a broadening can once again be attributed to the large spectral expansion undergone in the first pass of the amplifier.
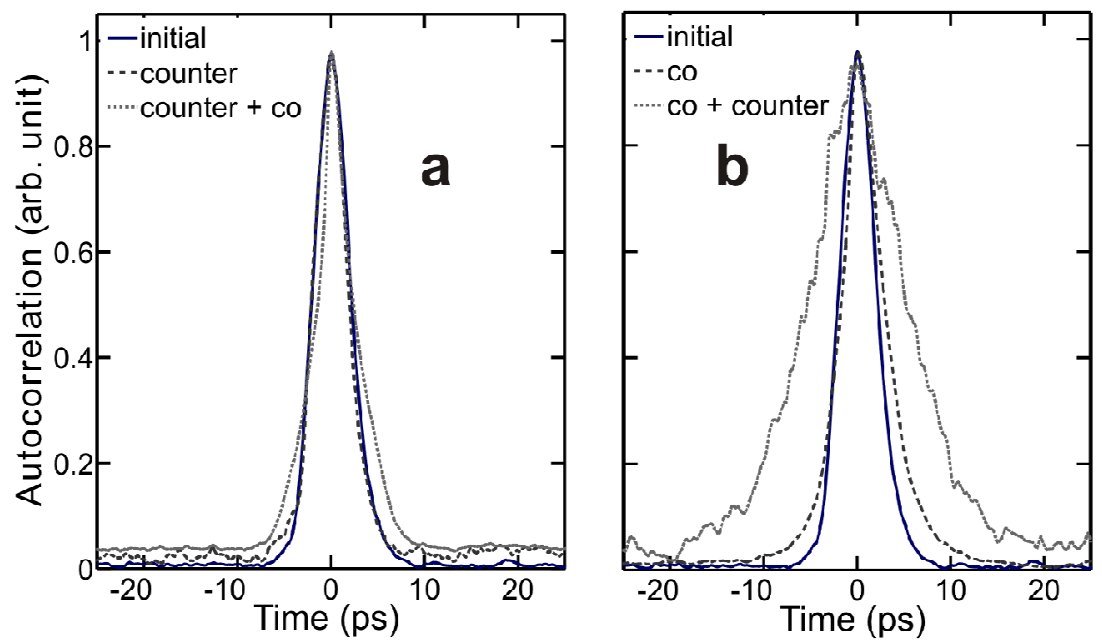

Figure 4: (a) Initial autocorrelation signal (blue solid line) is compared with autocorrelation for a counter-propagating configuration (dashed grey line) and for a counter-propagating pass followed by a co-propagating pass (dotted grey line). Results are compared to (b) autocorrelation for a co-propagating configuration (dashed grey line) and for a co-propagating pass followed by a counter-propagating pass (dotted grey line). Results obtained for an initial pump power of $1 \mathrm{~W}$.

We have also recorded the autocorrelation over a larger temporal span in order to observe the cross-correlation peaks between a pulse and its preceding pulse delayed by $100 \mathrm{ps}$. Such measurements were not possible in the previous published studies due to the low repetition rate of the passively modelocked source $[12,13,16]$ (requiring therefore a delay of several tens of ns). Interestingly, the level of this cross-correlation peak can provide pertinent information on the amplitude jitter degradation that affects the pulse train [25-27]. Experimental results are summarized in Fig. 5. The initial pulse train exhibits a level of the central peak identical to the level of the cross-correlation peak, confirming the regime of mode-locking of the source. Such low pulse-to-pulse amplitude jitter is maintained in a backward amplification scheme. On the contrary, when pump and signal co-propagate, the peak of cross-correlation falls drastically, 
which highlights the significant change of amplitude between two successive amplified pulses. This is fully consistent with the typical duration of the fluctuations of the pump as measured by autocorrelation measurements ( $25 \mathrm{ps,}$, see Fig. 1b), which is lower than the repetition rate that we considered. In this context, using higher repetition sources ( $80 \mathrm{GHz}$ or above) could provide some additional useful information: the typical duration ( $25 \mathrm{ps})$ of a high fluctuation of the Raman pump being shorter than the pulse separation (100 ps), our 10-GHz signal does not fully sample all the pump fluctuations and can be quite affected by the relative delay between the pump structure and the temporal pulse position $[16,19]$.

Using the temporal width of the cross-correlation peak, one could also evaluate the timing jitter [25]. We have found in our experiments that there was no significant increase of timing jitter in the amplification process, confirming therefore that the main source of degradation was amplitude jitter. Measurements carried out on dual pass configurations have shown that a similar signature of the high degradation of the amplitude stability is observed. Records made according to the pump power in a co-propagating configuration (Fig. 5c) confirm the conclusions drawn in section 3 (Fig. 3b). Indeed, increasing the pump power first leads to an increase of the number of extreme events, i.e. of the pulse-to-pulse fluctuations, translating into a lower cross-correlation peak. On the contrary, for higher gain, gain saturation reduces the fluctuations and the cross-correlation peak amplitude increases. 

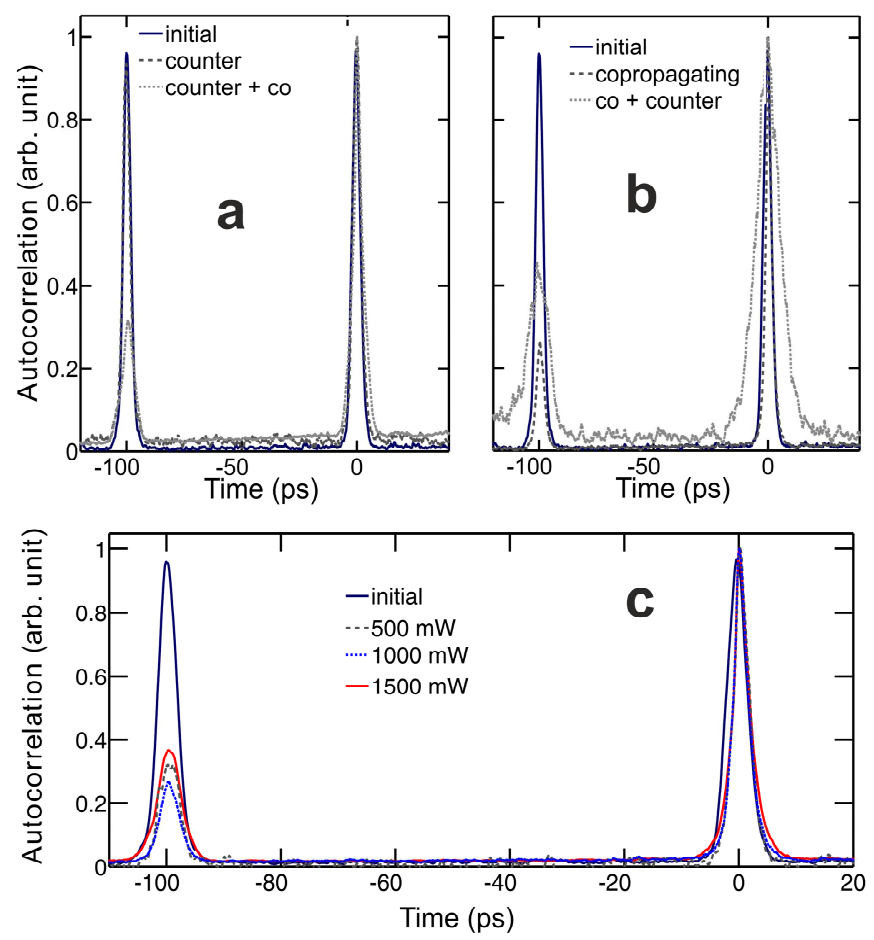

Figure 5: Cross-correlation showing signal degradation. (a) The initial cross-correlation (solid blue line) is compared with cross-correlation for a counter-propagating configuration (dashed grey line) and for a counter-propagating pass followed by a co-propagating pass (dotted grey line). Results are compared to (b) cross-correlation for a co-propagating configuration (dashed grey line) and for a co-propagating pass followed by a counter-propagating pass (dotted grey line). Results are obtained for an initial pump power of $1 \mathrm{~W}$. (c) Influence of the pump power in a co-propagating configuration for various initial pump powers: $500 \mathrm{~mW}$ (dashed grey line), $1 \mathrm{~W}$ (dotted blue line) and $1.5 \mathrm{~W}$ (solid red line).

\section{Radio frequency spectrum}

We complement our measurements by recording the radio frequency (RF) spectrum of the pulse train. Note that ideally, this data is linked to the autocorrelation signal we have previously described through the Wiener-Khinchine theorem. However, in practice, the finite temporal duration of analysis of the autocorrelator and the frequency bandwidth limitation of the optoelectronic of the usual radiofrequency spectrum analysers (as long as optical radiofrequency analysis is not used [28]) lead to a truncation of the signal, so that it is of interest to consider experimentally both records separately. Therefore, RF spectra are intensively used to 
characterize the optical amplifier properties: the measurement of the relative intensity noise (RIN) has indeed become a standard analysis to discuss the performance of optical amplifiers [18]. Other authors have also proposed to extract from the comb-like RF spectrum the amplitude and timing jitters of the periodic signal [29]. However, this latest application is not relevant to our experiment as it requires the analysis of a large number of harmonics peaks (we are limited by the RF bandwidth to the analysis of two $10 \mathrm{GHz}$ harmonic components) and as it is based on the strong assumption of small variations having a Gaussian probability distribution, which is clearly not our case in the described experiments.

In the context of this work, modestly, we show that depending on the configuration, the noise level significantly changes (Fig. 6). As predicted by the theoretical work of Fludger [30], we check that the backward pumping scheme exhibits significantly better performance than the forward pumping. What is striking on the signal obtained in this latest configuration is the presence of clear additional peaks in the vicinity of the $10 \mathrm{GHz}$ components. Those peaks are attributed to the transfer of intensity noise from the pumping laser to the pulsed signals and the use of a high-repetition rate $(10 \mathrm{GHz}$ instead of a few tens of $\mathrm{MHz}$ typical of modelocked sources) has facilitated their unambiguous detection. Note that these peaks are already present in the initial pump (Fig 6c) and are characteristic of the pumping laser used [31, 32]. The modal properties of this Raman fiber laser are quite complex and depend on the output power as can be seen from Fig. 6 (c): for output power of $1 \mathrm{~W}$, the noise is structured with spikes spaced by $0.6 \mathrm{GHz}$ whereas for $500 \mathrm{~mW}$, spikes are randomly spaced up to $0.5 \mathrm{GHz}$. Detailed study of the properties of the pumping laser exceed by far the scope of the present work, but these RF measurements suggest that the simplified assumptions made for modeling the behavior of the 
Raman amplifier (Gaussian optical spectrum with a random phase $[13,17,20]$ ) are not fully sufficient to retrieve the details of the RF spectrum.

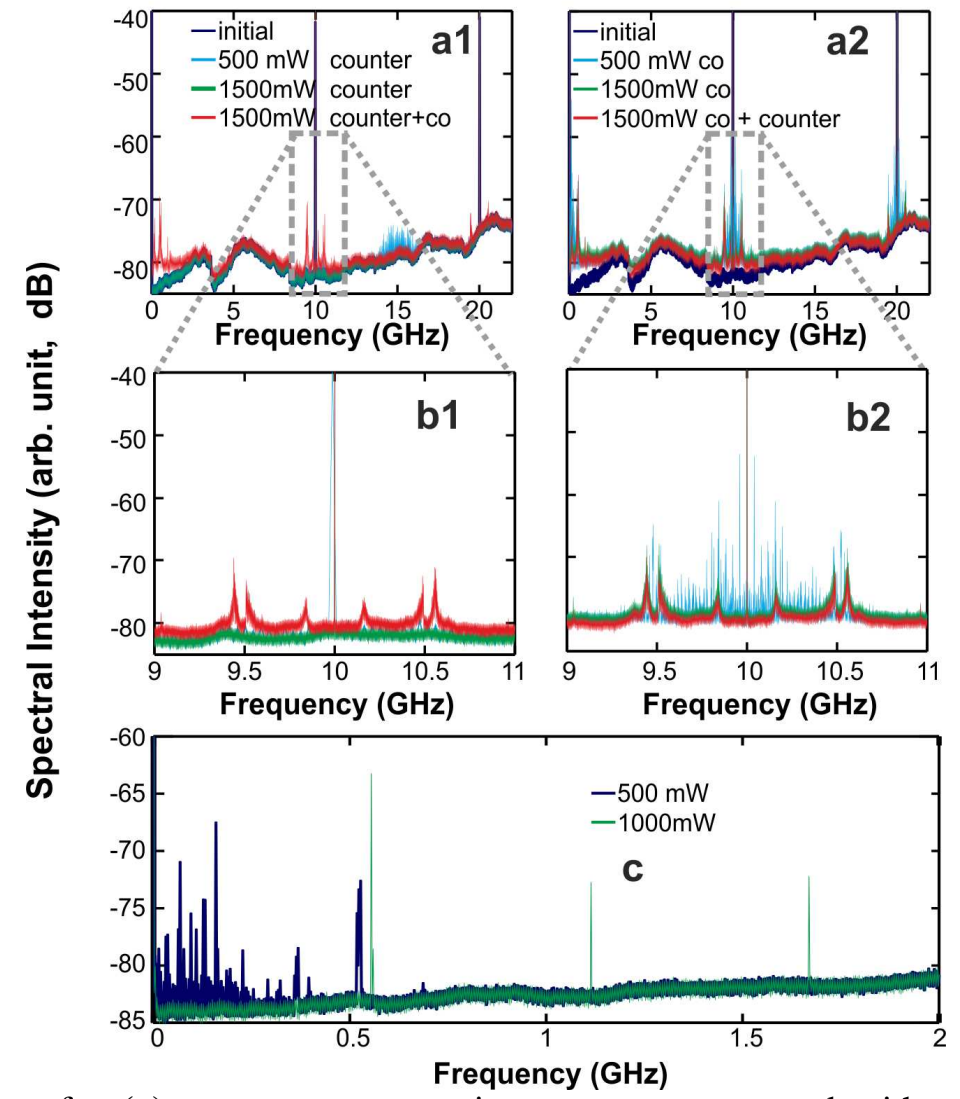

Figure 6: RF spectra for (a) a counter-propagating case are compared with spectra for (b) a copropagating case. In both cases, the initial RF spectrum of $10 \mathrm{GHz}$-laser (solid dark blue line) is compared to RF spectra obtained for an initial pump power of $500 \mathrm{~mW}$ (solid light blue line), $1500 \mathrm{~mW}$ (solid green line) and $1500 \mathrm{~mW}$ in dual-pass configuration (solid red line). Subplots (a2) and (b2) magnify the central part of spectra (between 9 and $11 \mathrm{GHz}$ ) respectively in (a1) and (b1). Results are compared to (c) RF spectra of the pump for two initial powers: $500 \mathrm{~mW}$ (solid dark blue line) and 1000 $\mathrm{mW}$ (solid green line) over $2 \mathrm{GHz}$.

\section{Optical Spectrum}

We also performed a spectral analysis by recording the output optical spectra. Indeed, we expect the rogue events to also significantly alter the phase of the pulses, which cannot be easily monitored using the previously described methods. Noticeable changes affecting the optical spectrum are summarized in Fig. 7. Two frequency spans were considered: a $150 \mathrm{~nm}$ span in 
order to observe the spectral broadening of the pulses and the cascading of the Raman process and a $5 \mathrm{~nm}$ span in order to observe the details of the spectral structure of the optical field.

From the 150-nm span records, we can first make out the significant spectral expansion that is experienced by the pulse train during its amplification in all the various pumping schemes. However, the level of spectral broadening differs significantly according to the setup. Indeed the broadening is increased for a co-propagating configuration, as a consequence of the longitudinal peak-power evolution over the fiber amplifier resulting for a forward pumping in a higher integrated self-phase modulation $[24,33]$. The impact of cross-phase modulation of the pump on the signal may also in a lesser extend increase the spectral broadening [16]. We have checked that filtering with a frequency offset optical band pass filter may offer an interesting way to isolate rarest and most intense pulse obtained in a co-propagating scheme [16]. On the contrary, for pulses amplified in a back-pumping scheme, the pulse train obtained after the same offset spectral filtering remains periodic with low peak-to-peak fluctuations.

We can also note a strong impact on the cascading of the Raman process. Contrary to a counter-propagating configuration where for a single pass amplifier, no cascading is observed, we can notice the emergence of a Stokes in a forward pumping scheme: the most powerful rogue structures have reached a peak power high enough to generate their own Raman Stokes. We therefore anticipate that filtering this Raman Stokes may offer an alternate solution to isolate rogue events. Such a Stokes wave may further be amplified in a second pass counter-propagating segment. 

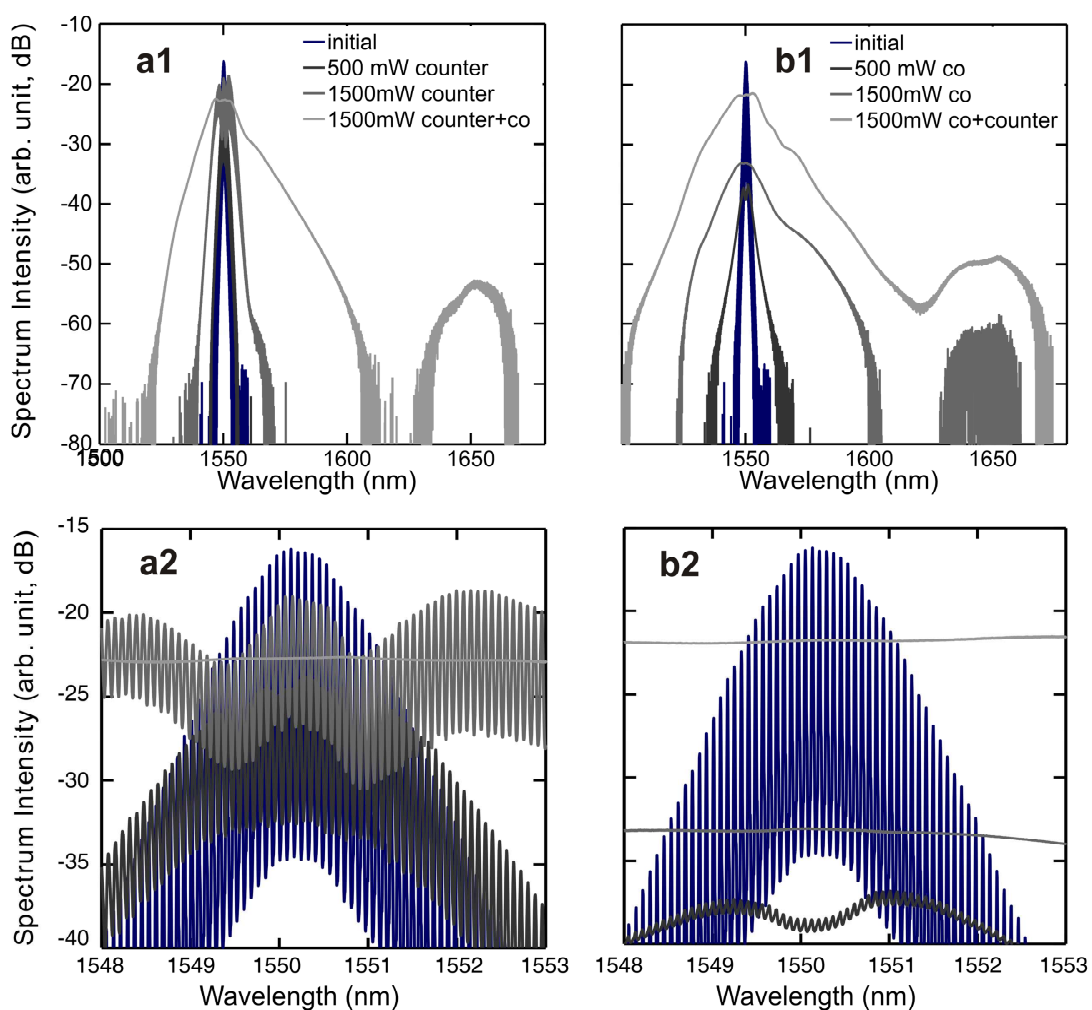

Figure 7: Optical Spectra for (a) a counter-propagating configuration and (b) a co-propagating configuration. In both cases, the initial spectrum of $10 \mathrm{GHz}$-laser (solid blue line) is compared to spectra obtained for an initial pump power of $500 \mathrm{~mW}$ (solid black line), $1500 \mathrm{~mW}$ (solid grey line) and $1500 \mathrm{~mW}$ in dual-pass configuration (solid light grey line). Subplots (a2) and (b2) magnify the central part of spectra respectively in (a1) and (b1).

Regarding the closer view of the central part of the spectra, we should ideally obtain a spectrum of lines spaced by the repetition rate, i.e. $10 \mathrm{GHz}$. Our initial spectrum (see Fig. 7) clearly exhibits such a comb structure although we are limited in some extent by the finite resolution $(0.02 \mathrm{~nm})$ of our optical spectrum analyzer (OSA). Note that the resolution used in this setup does not match the resolution with which OSNR measurements are usually performed in the optical domain [18], but such measure is not our aim here. The comb feature is maintained during the propagation in a counter-propagating configuration (Fig 7a2). However, we find that in a forward pumping scheme, the contrast of line spectrum falls dramatically (Fig $7 \mathrm{~b}$ ) and one can hardly guess the initial $10-\mathrm{GHz}$ lines. This indicates clearly the existence of significant 
fluctuations which breaks the $10-\mathrm{GHz}$ periodicity of the optical field. We note also that in a dualpass configuration, due to the changes in the co-propagating segment, the contrast is very poor.

\section{Spectrum observations based on wavelength-to-time mapping}

The optical spectra presented in the previous section are intrinsically averaged so that they cannot provide any information on the pulse-to-pulse spectral fluctuations. In order to get some insights of the spectral stability, we have added before detection on the photodiode a segment of dispersion compensating fiber (DCF with a dispersion of $-100 \mathrm{ps} / \mathrm{km} / \mathrm{nm}$ ). The amplified signal under investigation has been attenuated so that the linear propagation essentially imposes a large parabolic spectral phase. The length of the DCF fiber has been chosen so that the dispersionbroadened pulses do not overlap with the neighboring pulses, the 100 ps pulse spacing therefore represents a limit of the temporal broadening we induce.

The interest of this additional highly dispersive segment lies in the possibility it offers to translate the spectral shape into the temporal domain, which is a technique known as time stretch Fourier transform [34]: when the pulses are sufficiently dispersed, one can benefit from a one-toone mapping of the optical frequency into time. In other words, the fluctuations of the optical spectrum will be converted into fluctuations in the temporal domain and the eye diagram will enable to easily monitor those fluctuations. Note here that we focus exclusively on the eye diagram without trying to see each pulse individually. In this sense, we're going less far than B. Jalali and coworkers who have initially proposed this technique in order record individually optical rogue events $[1,34]$. Let us also stress that the configuration we study should not be confused with the Raman amplification scheme used to improve the sensitivity of the time stretch Fourier transform [35]. 

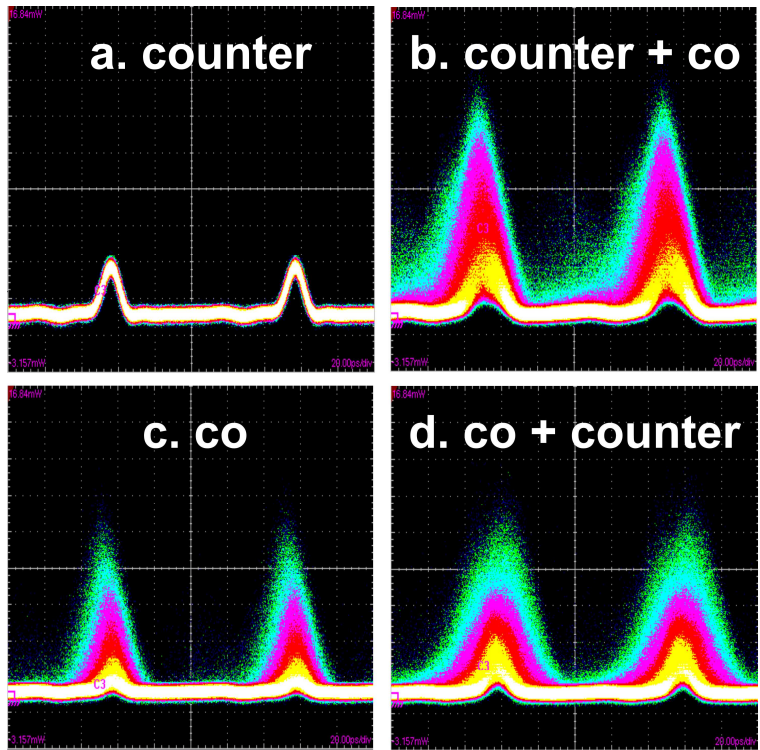

Figure 8: Eye diagrams for several configurations: (a) counter-propagating, (b) Dual pass starting by a counter-propagating pass (c) co-propagating and (d) Dual pass starting by a co-propagating pass. Results obtained for an initial pump power of $1.5 \mathrm{~W}$.

Results are summarized in Fig. 8 for the different pumping configurations. In the backward pumping scheme (Fig. 8a), stability is conserved, in agreement with the previously discussed results. In the other pumping configurations (Fig. 8 (b-d)), very large fluctuations of the temporal width are recorded revealing the dramatic pulse-to-pulse changes of the spectral extent of the pulses before this wavelength-time mapping process. This therefore confirms the interest of carrying spectral filtering with a frequency offset optical band pass filter to isolate the rarest and most intense pulses [16].

\section{Conclusions}

We have presented in this paper several non-standard characterization methods which highlight qualitatively the specific nature of fluctuations observed in a lumped Raman amplifier pumped in 
a co-propagating way. Similar events may also be found in other devices like waveguides as it has been shown by Borlaug et al. [36]. All the various effects highlighted here are mainly related to the transfer of pump noise on the amplified signal. In other words, there are not linked to accumulation of amplified spontaneous emission in the amplifier nor by the beat noise-noise or noise-signal nor by multi-interference pattern. Therefore, several widely-spread methods used to perform optical performance monitoring and determine optical signal to noise ratio [37] may fail to efficiently take into account all those highly damaging impairments.

All the trends described in this work have been confirmed by intensive numerical simulations (not detailed here) based the integration of a set of two coupled nonlinear Schrödinger equation including the Raman amplification $[13,16,17]$ with the assumption of a pump having a Gaussian statistics. Note however that this assumption fails in reproducing the results obtained by RF measurements, highlighting the complex nature of the output of the Raman fiber pump laser.

We expect our results to be expandable to other amplifiers such as parametric amplifiers. The physical principle of parametric amplifiers may however lead to additional points to be discussed such as the consequences of the phase-sensitive nature of those devices.

\section{Acknowledgements}

We would like to thank Guy Millot, Antonio Picozzi, Bertrand Kibler and Julien Fatome for fruitful discussions. This research was supported by the Agence Nationale de la Recherche (ANR 
MANUREVA project: ANR-08-SYSC-019) and by the Conseil Régional de Bourgogne (PARI Photcom). The experimental work has benefited from the PICASSO Platform equipment.

\section{References}

[1] D.R. Solli, C. Ropers, P. Koonath, B. Jalali, Optical rogue waves, Nature, 450 (2007) 1054.

[2] J.M. Dudley, C. Finot, G. Millot, J. Garnier, G. Genty, D. Agafontsev, F. Dias, Extreme events in optics: Challenges of the MANUREVA project, Eur. Phys. J. Special Topics, 185 (2010) 125-133.

[3] N. Akhmediev, A. Ankiewicz, M. Taki, Waves that appear from nowhere and disappear without a trace, Phys. Lett. A, 373 (2009) 675-678.

[4] B. Kibler, J. Fatome, C. Finot, G. Millot, F. Dias, G. Genty, N. Akhmediev, J.M. Dudley, The Peregrine soliton in nonlinear fibre optics, Nature Physics, 6 (2010) 790-795.

[5] M. Taki, A. Mussot, A. Kudlinski, E. Louvergneaux, M.I. Kolobov, M. Douay, Third-order dispersion for generating optical rogue solitons, Phys. Lett. A, 374 (2010) 691-695.

[6] K. Hammani, B. Kibler, C. Finot, A. Picozzi, Emergence of rogue waves from optical turbulence, Phys. Lett. A, 374 (2010) 3585-3589.

[7] A. Peleg, Log-normal distribution of pulse amplitudes due to Raman cross talk in wavelength division multiplexing soliton transmission, Optics Letters, 29 (2004) 1980-1982.

[8] Y.J. Chung, A. Peleg, Strongly non-Gaussian statistics of optical soliton parameters due to collisions in the presence of delayed Raman response, Nonlinearity, 18 (2005) 1555-1574.

[9] S. Vergeles, S.K. Turitsyn, Optical rogue waves in telecommunication data streams, Phys. Rev. A, 83 (2011) 061801.

[10] E.G. Turitsyna, G. Falkovich, V.K. Mezentsev, S.K. Turistyn, Optical turbulence and spectral condensate in long-fiber lasers, Phys. Rev. A, 80 (2009) 031804.

[11] D.V. Churkin, O. Gorbunov, S.V. Smirnov, Extreme value statistics in Raman fiber lasers, Opt. Lett., in press (2011).

[12] K. Hammani, C. Finot, G. Millot, Emergence of extreme events in fiber-based parametric processes driven by a partially incoherent wave, Opt. Lett., 34 (2009) 1138-1140.

[13] K. Hammani, C. Finot, J.M. Dudley, G. Millot, Optical rogue-wave fluctuations in fiber Raman amplifiers, Opt. Express, 16 (2008) 16467-16474.

[14] K. Hammani, C. Finot, B. Kibler, G. Millot, Soliton generation in a microstructured fiber by fourth order scalar modulation instability, IEEE Photon. J., 1 (2009) 205-212. 
[15] Y. Xu, S.G. Murdoch, Gain statistics of a fiber optical parametric amplifier with a temporally incoherent pump, Opt. Lett., (2010).

[16] C. Finot, K. Hammani, J. Fatome, J.M. Dudley, G. Millot, Selection of extreme events generated in Raman fiber amplifiers through spectral offset filtering, IEEE J. Quantum Electron., 46 (2009) 205-213.

[17] K. Hammani, C. Finot, A. Picozzi, Extreme statistics in Raman fiber amplifier : from analytical description to experiments, Opt. Commun., 284 (2010) 2594-2603.

[18] D.M. Baney, P. Gallion, R.S. Tucker, Theory and measurement techniques for the noise figure of optical amplifiers, Opt. Fiber. Technol., 6 (2000) 122-154.

[19] J. Schröder, S. Coen, Observation of high contrast, fast intensity noise of a continuous wave Raman fiber laser, Opt. Express, 17 (2009) 1644-1649.

[20] F. Vanholsbeeck, S. Martin-Lopez, M. Gonzalez-Herraez, S. Coen, The role of pump incoherence in continuous-wave supercontinuum generation, Opt. Express, 13 (2005) 66156625.

[21] C. Lafargue, J. Bolger, G. Genty, F. Dias, J.M. Dudley, B.J. Eggleton, Direct detection of optical rogue wave energy statistics in supercontinuum generation, Electronics Letters, 45 (2009) 217-218.

[22] P.A. Andrekson, M. Westlund, Nonlinear optical fiber based high resolution all-optical waveform sampling, Laser \& Photonics Rev., 1 (2007) 231-248.

[23] G.P. Agrawal, Nonlinear Fiber Optics, Fourth Edition, Academic Press, San Francisco, CA, 2006.

[24] C. Headley, G.P. Agrawal, Raman amplification in fiber optical communications, Academic Press, 2005.

[25] J. Fatome, J. Garnier, S. Pitois, M. Petit, G. Millot, M. Gay, B. Clouet, L. Bramerie, J.C. Simon, All-optical measurements of background, amplitude, and timing jitters for high speed pulse trains or PRBS sequences using autocorrelation function, Opt. Fiber. Technol., 14 (2007) 84-91.

[26] T.D. Vo, M.D. Pelusi, J. Schröder, F. Luan, S.J. Madden, D.-Y. Choi, D.A.P. Bulla, B. Luther-Davies, B.J. Eggleton, Simultaneous multi-impairment monitoring of $640 \mathrm{~Gb} / \mathrm{s}$ signals using photonic chip based RF spectrum analyzer, Opt. Express, 18 (2010) 3938-3945.

[27] M. Dinu, D.C. Kilper, H.R. Stuart, Optical performance monitoring using data stream intensity autocorrelation, J. Lightw. Technol., 24 (2006) 1194-1202.

[28] M. Pelusi, F. Luan, T.D. Vo, M.R.E. Lamont, S.J. Madden, D.A.P. Bulla, D.-Y. Choi, B. Luther-Davies, B.J. Eggleton, Photonic-chip-based radio-frequency spectrum analyser with terahertz bandwidth, Nat. Photonics, 3 (2009) 139-143.

[29] D. Von der Linde, Characterization of the noise in continuously operating mode-locked lasers, Appl. Phys. B, 39 (1986) 201-217.

[30] C. Fludger, V. Handerek, R.J. Mears, Pump to signal RIN transfer in Raman fiber amplifiers, J. Lightw. Technol., 19 (2001) 1140-1148. 
[31] S.A. Babin, V. Karalekas, P. Harper, E.V. Podivilov, V.K. Mezentsev, J.D. Ania-Castañón, S.K. Turitsyn, Experimental demonstration of mode structure in ultralong Raman fiber lasers, Opt. Lett., 32 (2007) 1135-1137.

[32] S.A. Babin, D.V. Churkin, A.A. Fotiadi, S.I. Kablukov, O.I. Medvedkov, E.V. Podivilov, Relative intensity noise in cascaded-Raman fiber lasers, IEEE Photon. Technol. Lett., 17 (2005) 2553-2555.

[33] C. Finot, Influence of the pumping configuration on the generation of optical similaritons in optical fibers, Opt. Commun., 249 (2005) 553-561.

[34] B. Jalali, D.R. Solli, K. Goda, K. Tsia, C. Ropers, Real-time measurements, rare events and photon economics, Eur. Phys. J. Special Topics, 185 (2010) 145-157.

[35] H. Chou, D.R. Solli, B. Jalali, Real-time spectroscopy with subgigahertz resolution using amplified dispersive Fourier transformation, Appl. Phys. Lett., 92 (2008) 111102.

[36] D. Borlaug, S. Fathpour, B. Jalali, Extreme value statistics in silicon photonics, IEEE Photon. J., 1 (2008) 33-39.

[37] Z. Pan, C. Yu, A.E. Willner, Optical performance monitoring for the next generation optical communication networks, Opt. Fiber. Technol., 16 (2010) 20-45. 